\title{
A RATIONAL SPECTRAL COLLOCATION METHOD WITH ADAPTIVELY TRANSFORMED CHEBYSHEV GRID POINTS
}

\author{
T. W. TEE* AND L. N. TREFETHEN ${ }^{\dagger}$
}

\begin{abstract}
A spectral collocation method based on rational interpolants and adaptive grid points is presented. The rational interpolants approximate analytic functions with exponential accuracy by using prescribed barycentric weights and transformed Chebyshev points. The locations of the grid points are adapted to singularities of the underlying solution, and the locations of these singularities are approximated by the locations of poles of Chebyshev-Padé approximants. Numerical experiments on two time-dependent problems, one with finite time blow-up and one with a moving front, indicate that the method far outperforms the standard Chebyshev spectral collocation method for problems whose solutions have singularities in the complex plane close to $[-1,1]$.
\end{abstract}

Key words. rational spectral collocation method, adaptive grid points, barycentric interpolation formula, complex singularities, Chebyshev-Padé approximation, blow-up problem, Burgers equation

AMS subject classifications. 65M70, 65M50, 41A20, 41A21

1. Introduction. The Chebyshev spectral method (the phrase spectral method is synonymous with spectral collocation method in this article) is a method for the numerical solution of differential equations on a bounded nonperiodic interval, which may be assumed without loss of generality to be $[-1,1]$. The basis of the method is to approximate the solution of a differential equation by a polynomial which interpolates data $u_{k}$ at Chebyshev points $x_{k}=\cos (k \pi / N), k=0,1, \ldots, N$, where the data $\left\{u_{k}\right\}$ are determined by requiring that the polynomial interpolant satisfy the differential equation exactly at the points $\left\{x_{k}\right\}$. As $N$ increases, the approximation error decays at a rate which depends on the smoothness of the underlying solution. If the solution can be continued as an analytic function in a closed ellipse with foci \pm 1 , semimajor axis length $L$, and semiminor axis length $l$, then the error decays exponentially at the rate $O\left((L+l)^{-N}\right)$ [24]. However, if the solution has singularities in the complex plane close to $[-1,1]$, so that $L+l \approx 1$, then this convergence rate will be too slow for the method to be effective.

One cannot simply alter the points and expect a better convergence rate, because polynomials which interpolate at Chebyshev points already give near best polynomial approximations. Instead, one must consider spectral methods based on other types of global interpolants, such as rational functions. Unlike polynomials, rational functions can interpolate at any set of points without the drawback of the Runge phenomenon.

The purpose of this article is to present a new spectral method based on rational interpolants and adaptive grid points, and to show that this method far outperforms the Chebyshev spectral method for problems whose solutions have singularities in the complex plane close to $[-1,1]$.

Spectral methods based on rational interpolants have been developed by Berrut and his collaborators [8]. A common feature of their methods is the representation of rational interpolants in barycentric form. The barycentric form of a rational function

* Oxford University Computing Laboratory, Wolfson Building, Parks Road, Oxford OX1 3QD, United Kingdom (wynn.tee@comlab.ox.ac.uk).

${ }^{\dagger}$ Oxford University Computing Laboratory, Wolfson Building, Parks Road, Oxford OX1 3QD, United Kingdom (LNT@comlab.ox.ac.uk). 
$r$ which interpolates data $f_{0}, f_{1}, \ldots, f_{N}$ at points $x_{0}, x_{1}, \ldots, x_{N}$ is

$$
r(x)=\frac{\sum_{k=0}^{N} \frac{w_{k}}{x-x_{k}} f_{k}}{\sum_{k=0}^{N} \frac{w_{k}}{x-x_{k}}},
$$

where $w_{0}, w_{1}, \ldots, w_{N}$ are called barycentric weights. An advantage of representing a rational interpolant in barycentric form is that its derivatives can be evaluated easily using differentiation formulae derived by Schneider and Werner [22], instead of using the quotient rule repeatedly. The $p$ th derivative of $r$ evaluated at $x_{j}$ can be written in the form $r^{(p)}\left(x_{j}\right)=\sum_{k=0}^{N} D_{j k}^{(p)} u_{k}$, where the entries of $D^{(1)}$ and $D^{(2)}$, the first and second order differentiation matrices, are given by

$$
\begin{gathered}
D_{j k}^{(1)}= \begin{cases}\frac{w_{k}}{w_{j}\left(x_{j}-x_{k}\right)}, & \text { if } j \neq k, \\
-\sum_{i=0, i \neq k}^{N} D_{j i}^{(1)}, & \text { if } j=k,\end{cases} \\
D_{j k}^{(2)}= \begin{cases}2 D_{j k}^{(1)}\left(D_{j j}^{(1)}-\frac{1}{x_{j}-x_{k}}\right), & \text { if } j \neq k, \\
-\sum_{i=0, i \neq k}^{N} D_{j i}^{(2)}, & \text { if } j=k,\end{cases}
\end{gathered}
$$

for $j, k=0,1, \ldots, N$. For every set of points $\left\{x_{k}\right\}$, there is a unique set of barycentric weights $\left\{w_{k}\right\}$, up to a multiplicative constant, for which (1.1) represents a polynomial interpolant. For example, if $\left\{x_{k}\right\}$ are the Chebyshev points, then (1.1) represents a polynomial interpolant if and only if $w_{0}=c / 2, w_{k}=(-1)^{k} c, k=1, \ldots, N-1$, and $w_{N}=(-1)^{N} c / 2$, for some constant $c$ [13]. Therefore, a polynomial interpolant can be made into a rational interpolant by modifying its points and leaving its barycentric weights unchanged, or vice versa.

Baltensperger, Berrut and Noël exploited this property, modifying a polynomial which interpolates at Chebyshev points into a rational interpolant which approximates analytic functions with exponential accuracy, by applying a conformal map to the points and leaving the barycentric weights unchanged [2]. Baltensperger and Berrut developed a spectral method based on such rational interpolants, in which they used the conformal map of Kosloff and Tal-Ezer [19] to distribute grid points more evenly over the computational interval $[1,7]$.

Berrut and Mittelmann took the alternative approach, modifying a polynomial which interpolates at Chebyshev points into a rational interpolant with preassigned poles, by multiplying the barycentric weights by some factors involving the poles and leaving the points unchanged [9]. They developed a spectral method based on such rational interpolants, in which they computed the locations of preasssigned poles by minimizing a residual error iteratively $[10,11]$.

Berrut and Mittelmann also developed a spectral method based on a combination of the two previous rational interpolants, in which they used a modified version of the conformal map of Bayliss and Turkel [6] to adapt grid points to multiple fronts, and 
computed the parameters of the map and the locations of poles simultaneously by minimizing a residual error iteratively [12]. However, this computationally expensive optimization step renders their method inefficient for time-dependent problems.

Spectral methods based on adaptive grid points have, so far, required that the underlying problem be transformed into new coordinates, where the transformation maps predefined grid points in a transformed coordinate into irregular grid points in a physical coordinate. The parameters of the transformation are usually chosen to minimize a smoothness functional $[4,5,17]$. Mulholland, Huang, and Sloan developed an adaptive spectral method, in which they equidistributed a monitor function using a finite difference method to locate adapted grid points in a physical coordinate, and constructed a transformation a posteriori from knowledge of the grid points in both transformed and physical coordinates [21].

In this article, we present a new adaptive rational spectral method which, unlike existing adaptive spectral methods, does not require that the underlying problem be transformed into new coordinates, and unlike existing rational spectral methods, takes into consideration singularities of the underlying solution. In $\S 2$, we explain how a conformal map which maps the singularities of a function farther into the complex plane can be used to improve the Chebyshev spectral method. In $\S 3$, we explain how Chebyshev-Padé approximation can be used to approximate the locations of these singularities. In $\S 4$, we explain how the new method can be implemented from ideas discussed in $\S 2$ and $\S 3$. In $\S 5$, we present the results of numerical experiments on two time-dependent problems. Finally in $\S 6$, we present some conclusions.

2. Enlarging the ellipse of analyticity. The basis of our adaptive rational spectral method is the following theorem, which was proved in [2], on the exponential convergence of rational functions which interpolate at transformed Chebyshev points.

Theorem 1. Let $D_{1}$ and $D_{2}$ be domains in $\mathbb{C}$ containing $J=[-1,1]$ and a real interval I respectively. Let $g: D_{1} \rightarrow D_{2}$ be a conformal map such that $g(J)=I$. If $f: D_{2} \rightarrow \mathbb{C}$ is such that $f \circ g: D_{1} \rightarrow \mathbb{C}$ is analytic inside and on an ellipse with foci \pm 1 , semimajor axis length $L$, and semiminor axis length $l$, then the rational function

$$
r_{N}(x)=\frac{\sum_{k=0}^{N} \frac{(-1)^{k}}{x-x_{k}} f\left(x_{k}\right)}{\sum_{k=0}^{N} \frac{(-1)^{k}}{x-x_{k}}}, \quad x_{k}=g(\cos (k \pi / N)),
$$

where the prime indicates that the $k=0$ and $k=N$ terms are halved, interpolates $f$ at the transformed Chebyshev points $\left\{x_{k}\right\}$ and satisfies

$$
\left|r_{N}(x)-f(x)\right|=O\left((L+l)^{-N}\right)
$$

uniformly for all $x \in[-1,1]$.

If the solution $u$ of a differential equation can be continued as an analytic function in a neighbourhood of $[-1,1]$ in the complex plane, then the error in approximating $u$ by the Chebyshev spectral method will decay exponentially at a rate which depends on the size of the largest ellipse with foci \pm 1 in which $u$ is analytic. To be brief, this largest ellipse will be called the ellipse of analyticity of $u$.

Theorem 1 suggests that one should choose a conformal map $g$ for which the ellipse of analyticity of $u \circ g$ is larger than the ellipse of analyticity of $u$, and apply $g$ in a spectral method based on rational interpolants of the form (2.1), to obtain an 
approximation of $u$ which is more accurate than that obtained using the Chebyshev spectral method with the same number of grid points. Alternatively, one can obtain an equally accurate approximation of $u$ by using the Chebyshev spectral method to approximate $u \circ g$ directly, but this approach requires the tedious transformation of the underlying differential equation into new coordinates. To be precise, if $x$ is the physical coordinate and $y=g^{-1}(x)$ is the transformed coordinate, then the first few derivatives of $u$ have to be transformed according to

$$
\begin{gathered}
\frac{d u}{d x}=\frac{1}{g^{\prime}(y)} \frac{d u}{d y}, \\
\frac{d^{2} u}{d x^{2}}=\frac{1}{\left[g^{\prime}(y)\right]^{2}} \frac{d^{2} u}{d y^{2}}-\frac{g^{\prime \prime}(y)}{\left[g^{\prime}(y)\right]^{3}} \frac{d u}{d y}, \\
\frac{d^{3} u}{d x^{3}}=\frac{1}{\left[g^{\prime}(y)\right]^{3}} \frac{d^{3} u}{d y^{3}}-\frac{3 g^{\prime \prime}(y)}{\left[g^{\prime}(y)\right]^{4}} \frac{d^{2} u}{d y^{2}}-\frac{g^{\prime \prime \prime}(y) g^{\prime}(y)-3\left[g^{\prime \prime}(y)\right]^{2}}{\left[g^{\prime}(y)\right]^{5}} \frac{d u}{d y},
\end{gathered}
$$

and similarly for higher derivatives. These transformations are not required when one approximates $u$ by a spectral method based on rational interpolants of the form (2.1).

How do we choose $g$ ? If only real solutions are of interest, then we should choose $g$ such that $g^{-1}$ maps the two complex conjugate singularities lying on the ellipse of analyticity of $u$ farther away from $[-1,1]$. There are many possibilities, but for the time being, consider the case where $u$ is analytic everywhere in the complex plane except along two lines extending from $0 \pm \varepsilon$ i to $0 \pm \infty \mathrm{i}$, as shown in the region $I_{1}$ of Figure 1. Then

1. $h_{1}(z)=-z \varepsilon^{-1} \mathrm{i}$ rotates and scales $I_{1}$ to produce $I_{2}$,

2. $h_{2}(z)=\sin ^{-1}(z)$ maps $I_{2}$ to the vertical strip $I_{3}$, and

3. $h_{3}(z)=-z / \sin ^{-1}\left(\varepsilon^{-1} \mathrm{i}\right)$ rotates and scales $I_{3}$ to produce $I_{4}$.

The conformal map $g$ which maps $I_{4}$ to $I_{1}$ is the inverse of $h_{3} \circ h_{2} \circ h_{1}$, and due to the identity $\sin ^{-1}(z)=-\mathrm{i} \sinh ^{-1}(\mathrm{i} z)$, can be written in the form

$$
g(z)=\varepsilon \sinh \left(z \sinh ^{-1}\left(\frac{1}{\varepsilon}\right)\right) .
$$

Figure 1 shows the adaptive nature of $g$, in that it maps points which are clustered near the boundaries of $[-1,1]$ into points which are clustered near the singular lines of $u$. Table 1 shows that as $\varepsilon$ decreases geometrically, the number of grid points required for the Chebyshev spectral method to approximate $u$ to 10 digits of accuracy increases geometrically, whereas for a spectral method based on rational interpolants of the form (2.1) and the conformal map (2.6), this number only increases algebraically.

The derivation of (2.6) can be generalized to cases where the two singular lines begin at $\delta \pm \varepsilon$ i instead of $0 \pm \varepsilon$ i. The conformal map $g$ becomes

$$
g(z)=\delta+\varepsilon \sinh \left[\left(\sinh ^{-1}\left(\frac{1-\delta}{\varepsilon}\right)+\sinh ^{-1}\left(\frac{1+\delta}{\varepsilon}\right)\right) \frac{z-1}{2}+\sinh ^{-1}\left(\frac{1-\delta}{\varepsilon}\right)\right] .
$$

The derivation of (2.7) makes no assumptions about the type of singularities of $u$, so (2.7) is applicable for all types of singularities, such as poles and branch points, as long as they are confined to the two singular lines. 

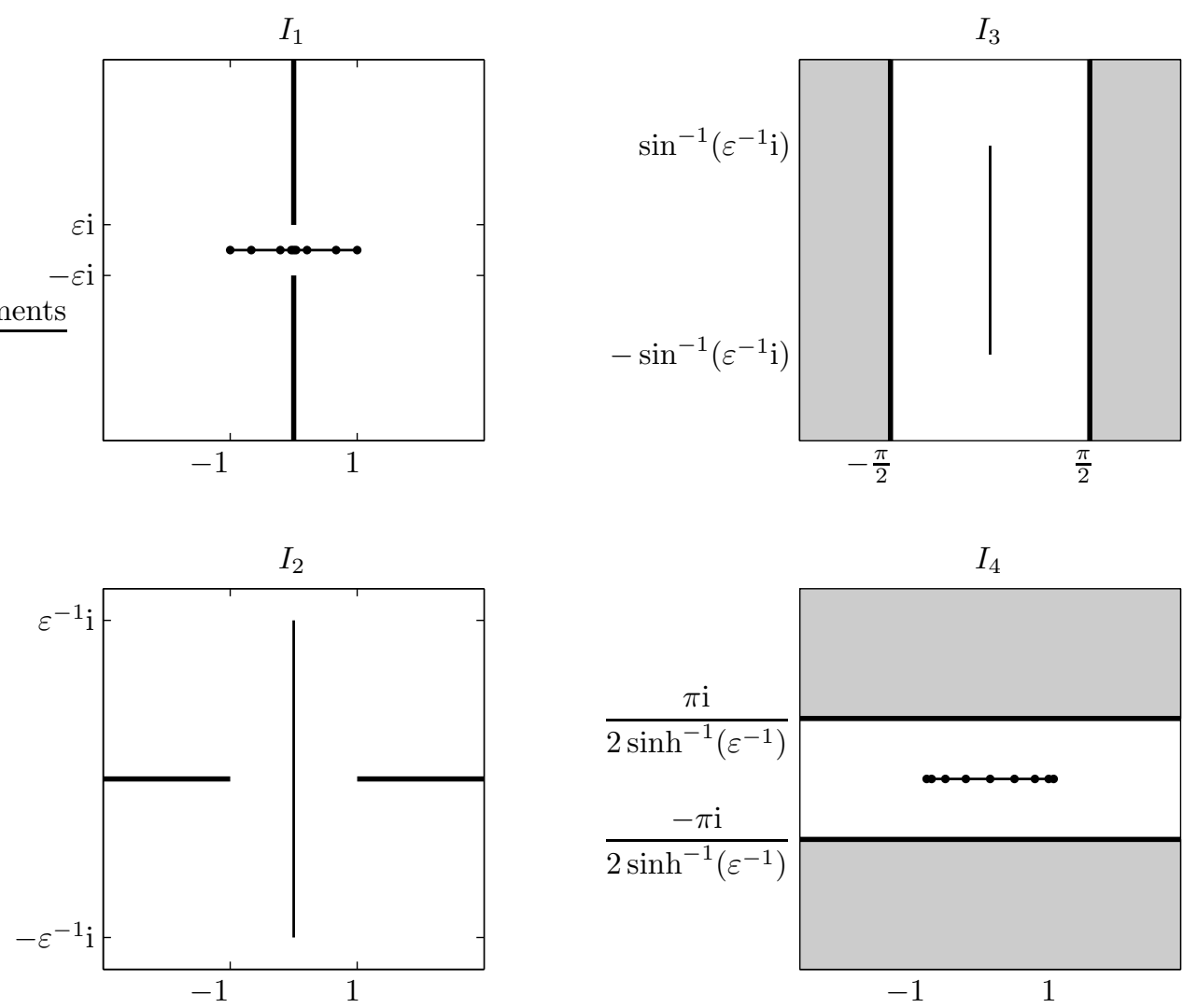

FIG. 1. Sequence of transformation images to be read from top to bottom, then from left to right. In the first panel, the thick lines represent singular lines, the thin line represents $[-1,1]$, and the white background represents the region of analyticity $I_{1}$. In the fourth panel, the dots represent Chebyshev points.

TABLE 1

Comparison of theoretical convergence rates for the Chebyshev spectral method and the adaptive rational spectral method. If a convergence rate is $O\left(K^{-N}\right)$, then $N_{\text {min }}$ is the smallest integer $N$ for which $K^{-N}$ is less than $10^{-10}$.

\begin{tabular}{|l|c|c|c|c|}
\hline \multirow{2}{*}{$\varepsilon$} & \multicolumn{2}{|c|}{ Chebyshev spectral method } & \multicolumn{2}{c|}{ Adaptive rational spectral method } \\
\cline { 2 - 5 } & Convergence rate & $N_{\min }$ & Convergence rate & $N_{\min }$ \\
\hline 1 & $O\left(2.4142^{-N}\right)$ & 27 & $O\left(3.8258^{-N}\right)$ & 18 \\
0.1 & $O\left(1.1050^{-N}\right)$ & 231 & $O\left(1.6528^{-N}\right)$ & 46 \\
0.01 & $O\left(1.0100^{-N}\right)$ & 2303 & $O\left(1.3395^{-N}\right)$ & 79 \\
0.001 & $O\left(1.0010^{-N}\right)$ & 23026 & $O\left(1.2277^{-N}\right)$ & 113 \\
0.0001 & $O\left(1.0001^{-N}\right)$ & 230259 & $O\left(1.1711^{-N}\right)$ & 146 \\
\hline
\end{tabular}

3. Locating the singularities. The parameters $\delta$ and $\varepsilon$ required in (2.7) are usually not known analytically, so it is necessary to approximate them using some singularity location technique. The technique must be computationally cheap for our adaptive rational spectral method to be more efficient than existing rational spectral methods. The technique which we chose is based on Chebyshev-Padé approximation. 
It is conceptually similar to the technique presented by Weideman in [25], where the locations of singularities are approximated by the locations of poles of Fourier-Padé approximants.

If $f$ is a continuous function on $[-1,1]$, then it has a Chebyshev expansion

$$
f(x)=\sum_{k=0}^{\infty} a_{k} T_{k}(x),
$$

where $a_{k}=\frac{2}{\pi} \int_{-1}^{1}\left(1-x^{2}\right)^{-\frac{1}{2}} f(x) T_{k}(x) d x$ ( $a_{0}$ is half the value given by this formula) and $T_{k}(x)=\cos \left(k \cos ^{-1}(x)\right)$. The scalars $\left\{a_{k}\right\}$ are called Chebyshev coefficients and the functions $\left\{T_{k}\right\}$ are called Chebyshev polynomials. The linear $[m, n]$ ChebyshevPadé approximation of $f$ is defined as the rational function

$$
r_{m, n}(x) \equiv \frac{p(x)}{q(x)}=\frac{\sum_{k=0}^{m} b_{k} T_{k}(x)}{1+\sum_{k=1}^{n} c_{k} T_{k}(x)},
$$

of numerator degree at most $m$ and denominator degree at most $n$, such that the Chebyshev expansion of $f q-p$ satisfies

$$
f(x) q(x)-p(x)=O\left(T_{m+n+1}(x)\right) .
$$

The coefficients $\left\{b_{k}\right\}$ and $\left\{c_{k}\right\}$ can be determined by substituting (3.1) and (3.2) into (3.3), matching terms with Chebyshev polynomials of equal degree, and finally solving the system of linear equations

$$
\left(\begin{array}{cccccc}
1 & & & -d_{0,1} & \cdots & -d_{0, n} \\
& \ddots & & \vdots & \vdots & \vdots \\
& & 1 & -d_{m, 1} & \cdots & -d_{m, n} \\
& & & -d_{m+1,1} & \cdots & -d_{m+1, n} \\
& & & \vdots & \vdots & \vdots \\
& & & -d_{m+n, 1} & \cdots & -d_{m+n, n}
\end{array}\right)\left(\begin{array}{c}
b_{0} \\
\vdots \\
b_{m} \\
c_{1} \\
\vdots \\
c_{n}
\end{array}\right)=\left(\begin{array}{c}
a_{0} \\
\vdots \\
a_{m} \\
a_{m+1} \\
\vdots \\
a_{m+n}
\end{array}\right)
$$

where $\sum_{j=0}^{\infty} d_{j, k} T_{j}(x)=\sum_{j=0}^{\infty} a_{j} T_{j}(x) T_{k}(x)$, for $\left\{b_{k}\right\}$ and $\left\{c_{k}\right\}$ as required. It should be noted that $\left\{c_{k}\right\}$ can be computed independently of $\left\{b_{k}\right\}$, in which case the system of linear equations to be solved is only of size $n$. The poles of $r_{m, n}$ can be determined by computing the eigenvalues of the companion matrix $[3,14]$

$$
C=\left(\begin{array}{cccccc}
0 & 1 & & & & \\
\frac{1}{2} & 0 & \frac{1}{2} & & & \\
& \ddots & \ddots & \ddots & & \\
& & \ddots & \ddots & \ddots & \\
\frac{c_{0}}{-2 c_{n}} & \frac{c_{1}}{-2 c_{n}} & \cdots & \frac{c_{n-3}^{2}}{-2 c_{n}} & \frac{c_{n-2}}{-2 c_{n}}+\frac{1}{2} & \frac{c_{n-1}}{-2 c_{n}}
\end{array}\right),
$$

where $c_{0}=1$. This is a matrix eigenvalue problem of size $n$. It can be deduced from (3.4) that $r_{m, n}$ always exists and is unique provided that $c_{n} \neq 0$. 
How do we determine $\delta$ and $\varepsilon$ ? The aim is to approximate them by the real and imaginary parts of the poles of a Chebyshev-Padé approximant. If we can evaluate the solution $u$ of a differential equation at $M+1$ Chebyshev points, typically scaled and translated to a subinterval of $[-1,1]$ rather than $[-1,1]$ itself, then we can compute $M+1$ approximate Chebyshev coefficients of $u$ by applying the Fast Fourier Transform to the values of $u$ at these Chebyshev points. The poles of an $[m, 2]$ Chebyshev-Padé approximation of $u$, where $m$ is a small integer which we take to be $M / 2$ rounded to the nearest integer, can then be computed by applying the techniques just described. The locations of these poles approximate the locations of the two complex conjugate singularities lying on the ellipse of analyticity of $u$ [23], and hence $\delta$ and $\varepsilon$. The overall work involves solving a $2 \times 2$ system of linear equations and computing the eigenvalues of a $2 \times 2$ matrix, both of which are trivial computationally.

4. Implementation. How do we put together a spectral method based on the ideas discussed in $\S 2$ and $\S 3$ ? If we know the values of the solution $u$ of a differential equation at grid points $x_{0}^{n}, x_{1}^{n}, \ldots, x_{N}^{n}$ and time $t^{n}$, then we approximate $u$ by the rational interpolant

$$
r_{N}^{n}(x, t)=\frac{\sum_{k=0}^{N} \frac{(-1)^{k}}{x-x_{k}^{n}} r_{N}^{n}\left(x_{k}^{n}, t\right)}{\sum_{k=0}^{N} \frac{(-1)^{k}}{x-x_{k}^{n}}},
$$

where $r_{N}^{n}\left(x_{k}^{n}, t^{n}\right)=u\left(x_{k}^{n}, t^{n}\right)$. The data $\left\{r_{N}^{n}\left(x_{k}^{n}, t^{n+1}\right)\right\}$ approximating $\left\{u\left(x_{k}^{n}, t^{n+1}\right)\right\}$ can be determined by firstly discretizing the underlying differential equation using a temporal discretization method of choice, and secondly requiring that $r_{N}^{n}$ satisfy the discretized differential equation exactly at the grid points $\left\{x_{k}^{n}\right\}$, where the derivatives of $r_{N}^{n}$ can be evaluated as the product of differentiation matrices and data vectors, as mentioned in $\$ 1$. In MATLAB, the first and second order differentiation matrices can be constructed by calling bcmatrix:

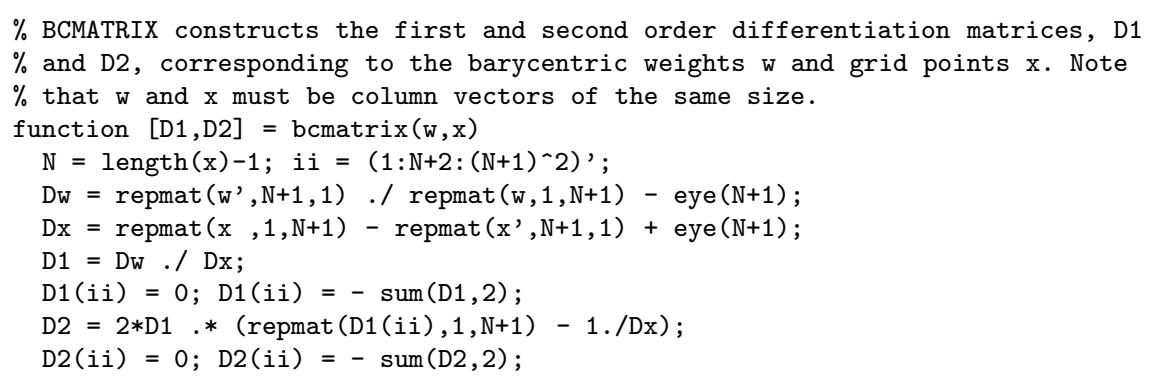

There is no difference between what we have done so far and what we would do with the Chebyshev spectral method. The difference arises when we adapt grid points for the next time step, for which we do the following.

Algorithm 1. At the end of the nth time step:

1. Evaluate $r_{N}^{n}\left(x, t^{n+1}\right)$ at $M+1$ Chebyshev points scaled and translated to a subinterval of $[-1,1]$ centered at $\delta$,

2. Compute approximate Chebyshev coefficients of $r_{N}^{n}\left(x, t^{n+1}\right)$ in that interval from the values in step 1 using the Fast Fourier Transform,

3. Compute new values of $\delta$ and $\varepsilon$ from the Chebyshev coefficients in step 2 using the Chebyshev-Padé singularity location technique, 
4. Define new grid points $x_{k}^{n+1}=g(\cos (k \pi / N)), k=0,1, \ldots, N$, where $g$ is given by (2.7) with the new $\delta$ and $\varepsilon$, and

5. Evaluate $r_{N}^{n}\left(x, t^{n+1}\right)$ at the new grid points $\left\{x_{k}^{n+1}\right\}$, and use these values as initial data for the next time step.

In practice, we start with a grid of Chebyshev points, and only begin adapting the grid points when $\varepsilon$ falls below a threshold indicating how close the true singularities of $u$ in the complex plane are to $[-1,1]$. In our numerical experiments, we take this threshold to be 0.5 . It is necessary to make a few remarks about steps $1-3$.

The number $M+1$ of Chebyshev points in step 1 should be enough for $r_{N}^{n}\left(x, t^{n+1}\right)$ to be approximated to a prescribed accuracy in the subinterval centered at $\delta$. The width of the subinterval should be sufficiently small for $M$ to be small too, and yet sufficiently large for the real part of the true singularities of $u\left(x, t^{n+1}\right)$ to lie within it. In our numerical experiments, we take $M$ to be the smallest power of two for which the Chebyshev coefficient of largest subindex in step 2 is less than $10^{-6}$ in absolute value, and we take the subinterval to be $[\delta-\zeta, \delta+\zeta]$, where $\zeta=\min (10 \varepsilon, 1-|\delta|)$. A typical value of $M$ is 128 . In MATLAB, step 1 (and step 5) can be implemented by calling bcinterp:

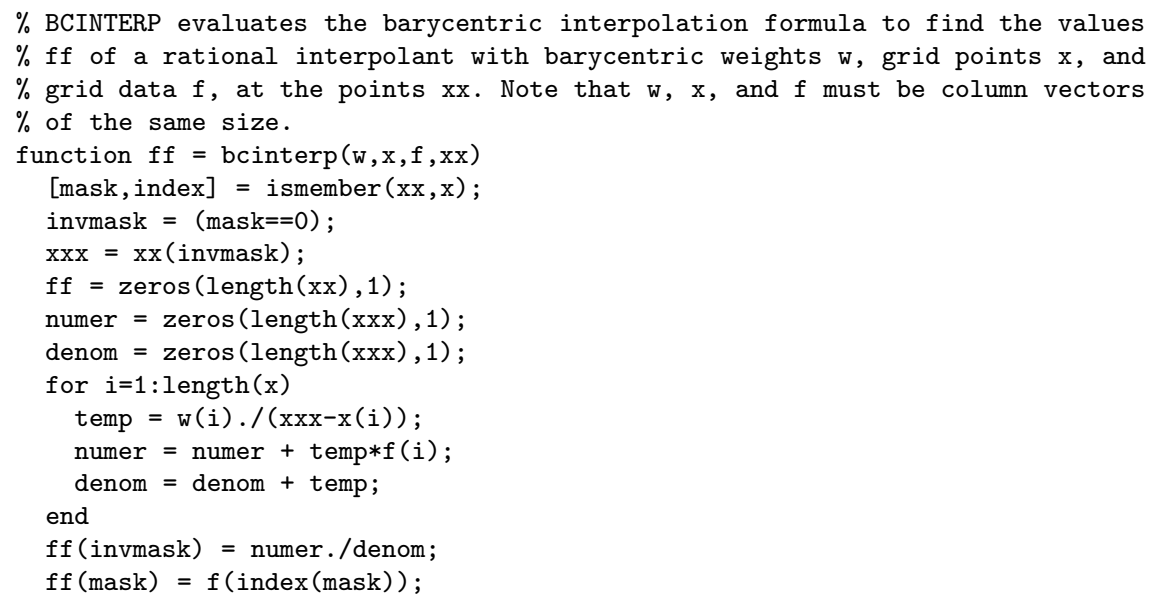

and step 2 can be implemented by calling chebpoly:

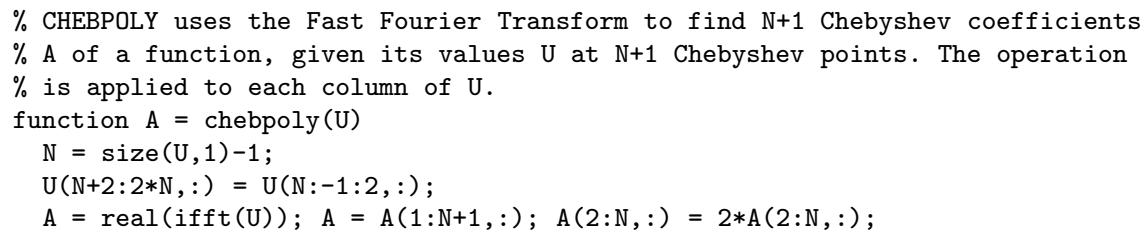

The new $\varepsilon$ in step 3 should be multiplied by a safety factor to ensure that (2.7) accounts for the true singularities of $u\left(x, t^{n+1}\right)$. In our numerical experiments, we take this safety factor to be 0.75 . In MATLAB, step 3 can be implemented by calling chebpade, which in turn calls chebeval and chebpoly:

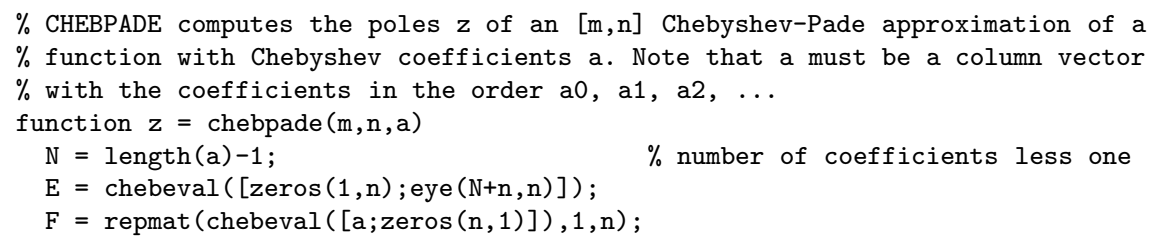




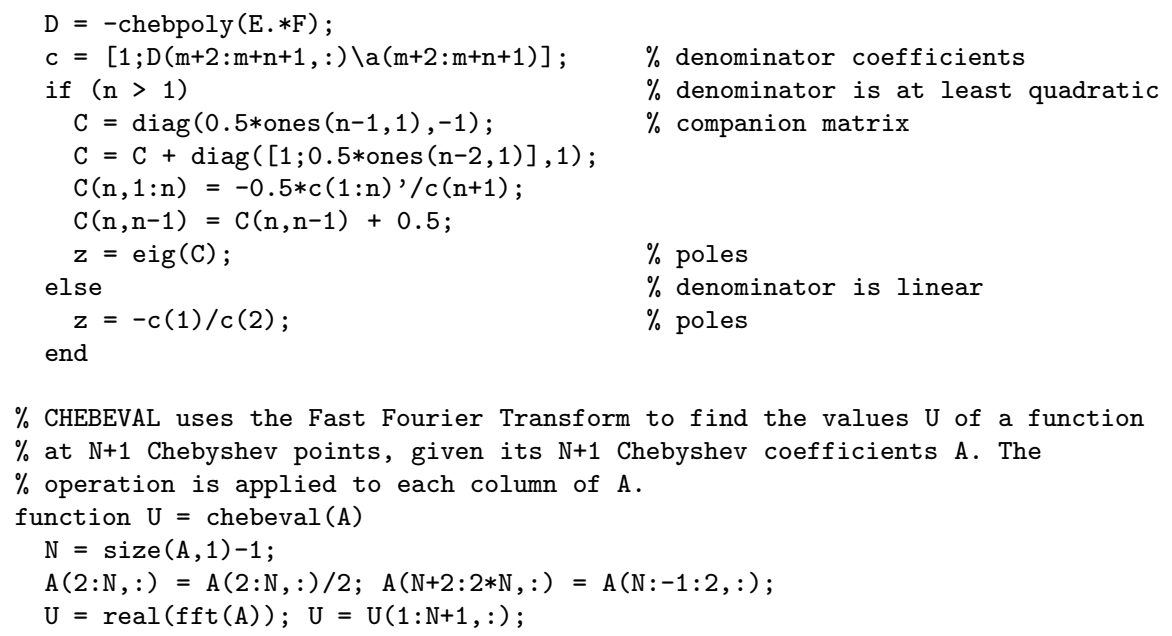

5. Numerical experiments. We now present the results of numerical experiments on two time-dependent problems. The problems are discretized temporally using the adaptive Runge-Kutta 5(4) method of Dormand and Prince [16] with an error tolerance of $10^{-10}$ (we did not use the ode45 solver of MATLAB, even though it implements the same method, because it does not give us the option of pausing at the end of each time step to adapt the grid points), and solutions are computed using the Chebyshev spectral method and our adaptive rational spectral method with various $N<128$. The approximation errors are calculated as the maximum absolute value of the difference between the various approximations and their $N=128$ counterparts evaluated at 10001 equispaced points.

5.1. Blow-up problem. The first problem is the Frank-Kamenetskii or Gelfand equation

$$
\frac{\partial u}{\partial t}=\frac{\partial^{2} u}{\partial x^{2}}+e^{u}, \quad-1<x<1,
$$

with the initial condition $u(x, 0)=0$ and boundary conditions $u(-1, t)=u(1, t)=0$. The equation arises in mathematical models of exothermic chemical reactions in solids [20]. The solution of this problem is symmetric with a peak at $x=0$ which blows up to infinity in finite time due to the nonlinear exponential term, a phenomenon known in the literature as thermal runaway. The solution has logarithmic singularities along the line $\operatorname{Re}(x)=0$ near the blow-up time $T$ [15], but this time is not known to high precision. The numerical experiment was run until $t=3.544664598$. The results are summarized in Figure 2.

The first row of the figure shows the solution $u$ approximated using the adaptive rational spectral method with $N=56$. The grid points are shown as dots. The logarithmic blow-up effect can be seen in the fact that when time moves forward by two digits, the height of $u$ increases by almost a fixed amount. The equidistribution of grid points along the approximation curves enables the method to resolve the tall sharp peak.

The second row shows the poles whose locations approximate the locations of the two complex conjugate singularities lying on the ellipse of analyticity of $u$. The poles appear along the line $\operatorname{Re}(x)=0$ in agreement with the theory. They move closer to each other as the peak grows taller, and coalesce at the blow-up time [25]. 

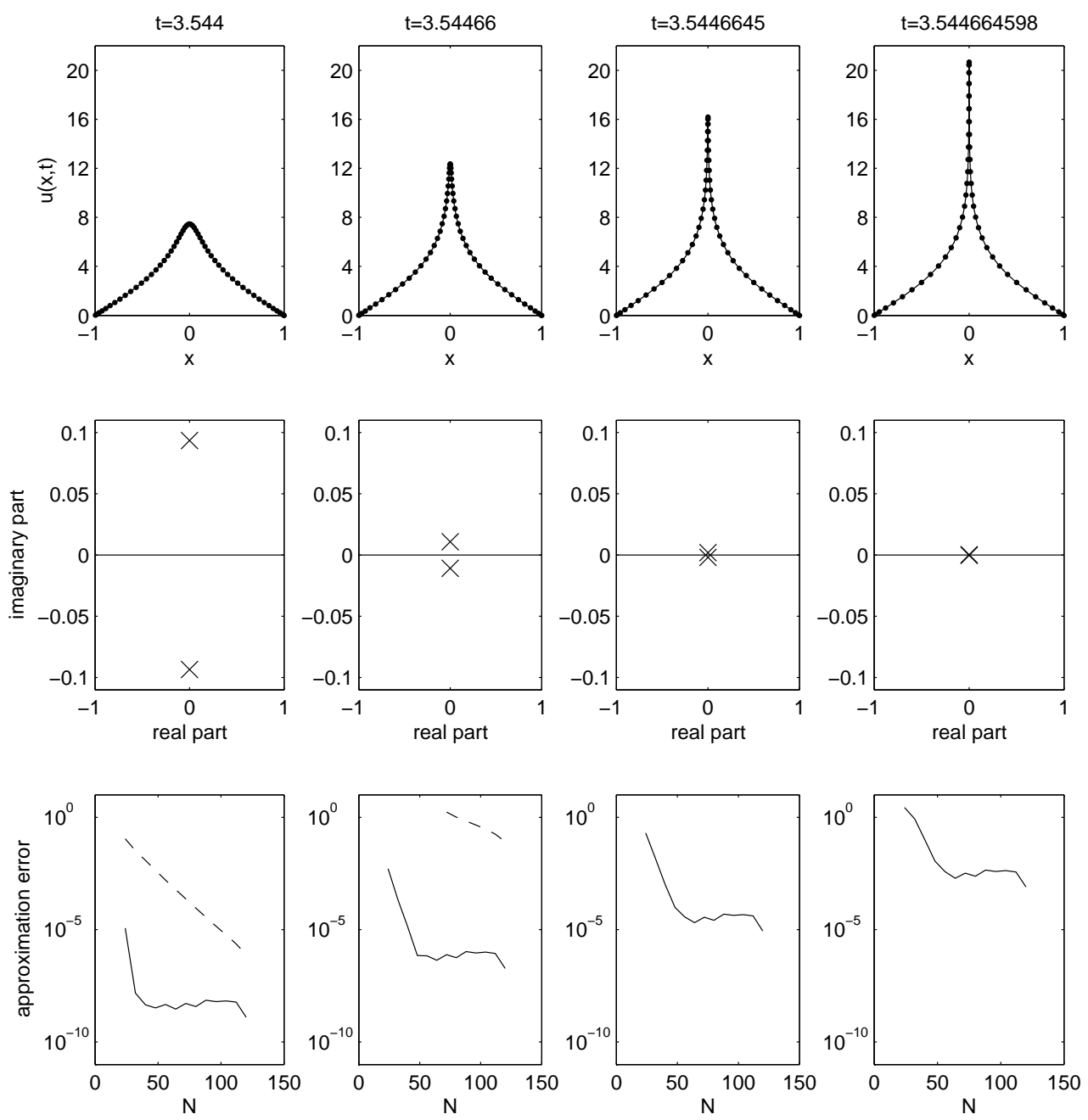

FIG. 2. The first row shows approximations of the solution of (5.1) computed using the adaptive rational spectral method with $N=56$. Dots represent grid points. The second row shows the poles computed using the Chebyshev-Padé singularity location technique. The third row shows log-linear plots of approximation error against $N$. Dashed lines correspond to the Chebyshev spectral method and solid lines to the adaptive rational spectral method. Dashed lines are absent where the Chebyshev spectral method failed to produce a result.

The third row shows plots of approximation error against $N$ on a log-linear scale for the Chebyshev spectral method and the adaptive rational spectral method. As the height of $u$ increases from about 8 to 20, the maximum number of accurate digits for the adaptive rational spectral method decreases in steps of about 2 , reflecting the loss of absolute accuracy in the evaluation of $e^{u}$ as its magnitude grows in steps of about 2 digits and its relative accuracy remains at about 16 digits. Apart from this quirk, the method requires about 50 grid points to produce results which are accurate to the limit of temporal errors. In contrast, the Chebyshev spectral method fails to produce any result beyond $t=3.54466$ for $N \leq 128$.

We also include Figure 3 which compares shapes of a computed approximation of 

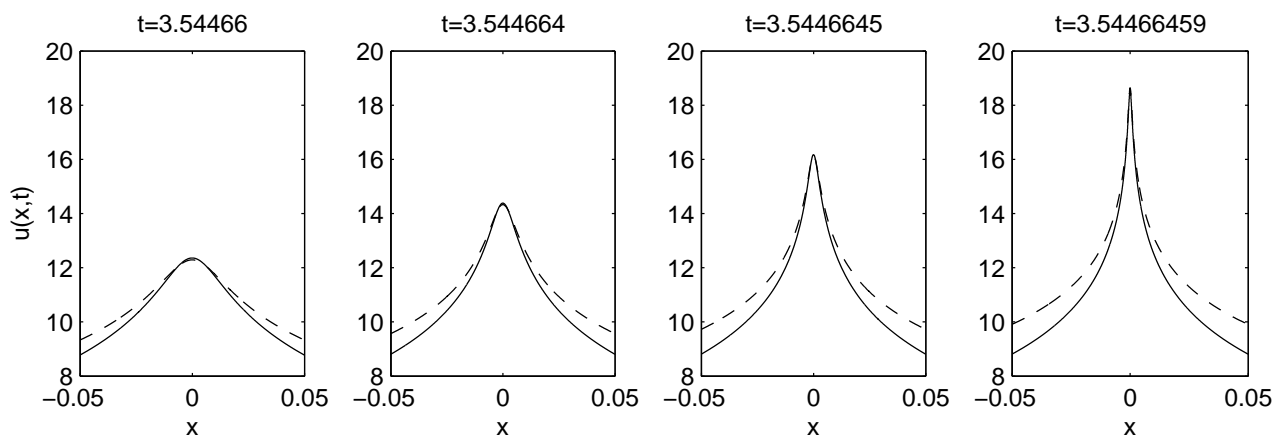

Fig. 3. Shapes of the solution of (5.1) near the blow-up point and blow-up time. Dashed curves correspond to the asymptotic approximation (5.2), and solid curves to the adaptive rational spectral method with $N=56$.

TABLE 2

The solution of (5.1) evaluated at $x=0$ approximated using the adaptive rational spectral method.

\begin{tabular}{|c|c|c|c|c|}
\hline$N$ & $t=3.544$ & $t=3.54466$ & $t=3.5446645$ & $t=3.544664598$ \\
\hline 32 & 7.460488435 & 12.36019369 & 16.16163618 & 19.81771341 \\
48 & 7.460488439 & 12.36040241 & 16.17636012 & 20.65913394 \\
64 & 7.460488439 & 12.36040263 & 16.17643386 & 20.66847337 \\
80 & 7.460488439 & 12.36040250 & 16.17642809 & 20.66802983 \\
96 & 7.460488436 & 12.36040214 & 16.17641126 & 20.66650626 \\
112 & 7.460488436 & 12.36040219 & 16.17641359 & 20.66671875 \\
\hline
\end{tabular}

$u$ near the blow-up time with the asymptotic approximation [15]

$$
u(x, t)=\xi-\log \left(1+\eta^{2}\right)-\frac{5}{2} \frac{\log (\xi)}{\xi} \frac{\eta^{2}}{1+\eta^{2}}+O\left(\frac{1}{\xi}\right)
$$

where $\xi=-\log (T-t), \eta=x / \sqrt{4 \xi(T-t)}$, and $T=3.544664598$. The shapes only match near $x=0$, because (5.2) is only valid as $x \rightarrow 0$ and $t \rightarrow T$. In addition, we include Table 2 which lists the height of $u$ approximated using the adaptive rational spectral method with various $N$, and hope that it will serve as a reference for further study of this blow-up problem.

5.2. Moving front problem. The second problem is the Burgers equation

$$
\frac{\partial u}{\partial t}=\nu \frac{\partial^{2} u}{\partial x^{2}}-u \frac{\partial u}{\partial x}, \quad 0<x<1
$$

with the initial condition $u(x, 0)=\sin (2 \pi x)+\frac{1}{2} \sin (\pi x)$ and boundary conditions $u(0, t)=u(1, t)=0$. This problem serves as a common test problem for adaptive grid methods (see [18] and the references therein). Its solution develops a steep front which moves towards $x=1$, but due to the homogeneous boundary conditions, also decays with time. The width of the front is $O(\nu)$. The numerical experiment was run until $t=0.90$ with $\nu=10^{-3}$. The results are summarized in Figure 4.

The first row of the figure shows the solution $u$ approximated using the adaptive rational spectral method with $N=56$. The grid points are shown as dots. The 

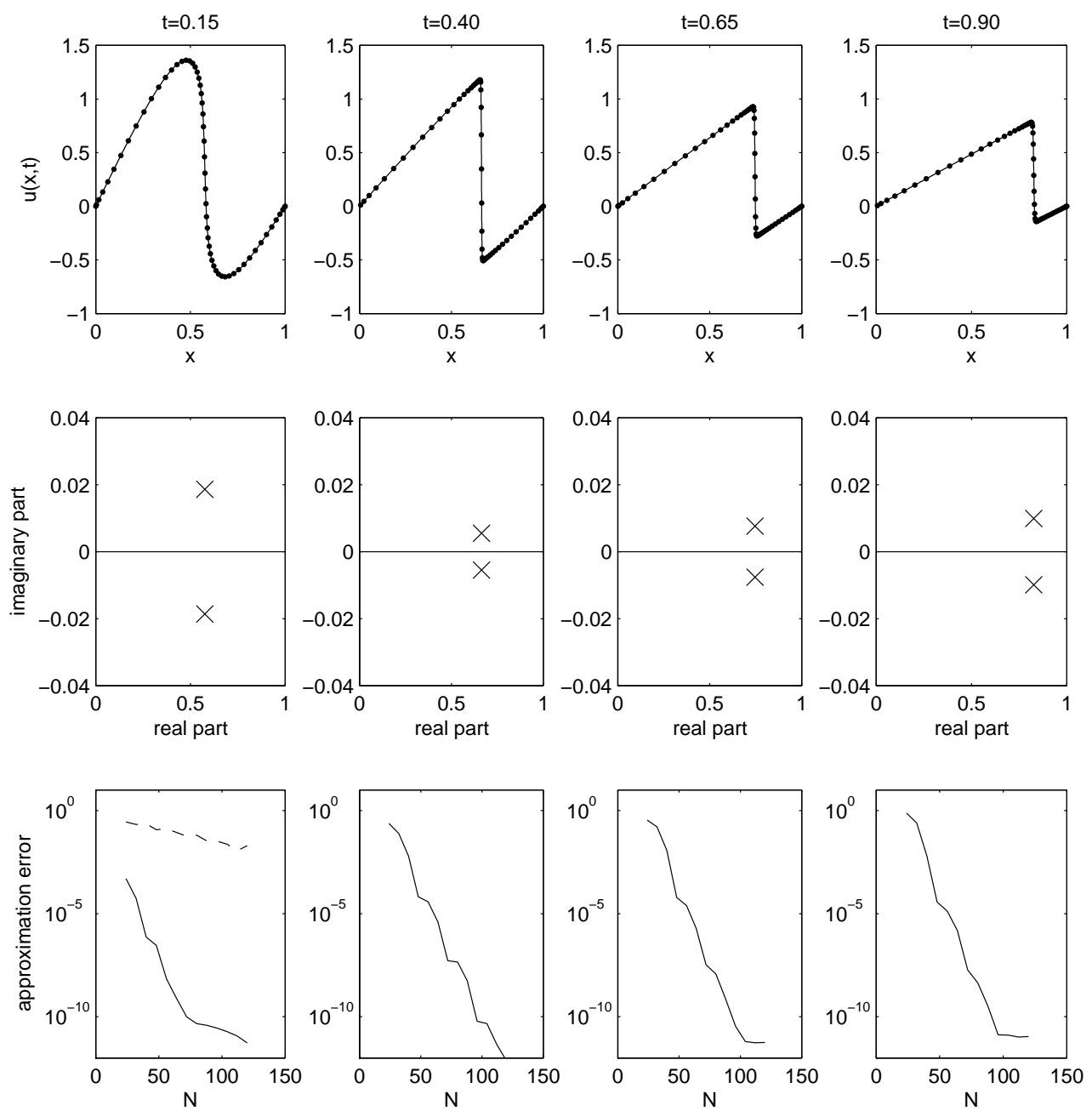

FIG. 4. Like Figure 2 but for (5.3) with $\nu=10^{-3}$.

clustering of grid points where the approximation curves have steep gradients enables the method to resolve the thin steep front.

The second row shows the poles whose locations approximate the locations of the two complex conjugate singularities lying on the ellipse of analyticity of $u$. The poles move closer to each other when the front steepens, and apart again when the front decays. They also move towards $\operatorname{Re}(x)=1$ in accordance with the front. In the absence of viscosity, they would coalesce when a shock is formed [25].

The third row shows plots of approximation error against $N$ on a log-linear scale for the Chebyshev spectral method and the adaptive rational spectral method. The latter requires about 100 grid points to produce results which are accurate to the limit of temporal errors. In contrast, the Chebyshev spectral method fails to produce any result beyond $t=0.15$ for $N \leq 128$.

We also varied $\nu$ and include Figure 5 which shows plots of approximation error against $N$ on a $\log$-linear scale for the adaptive rational spectral method with various 

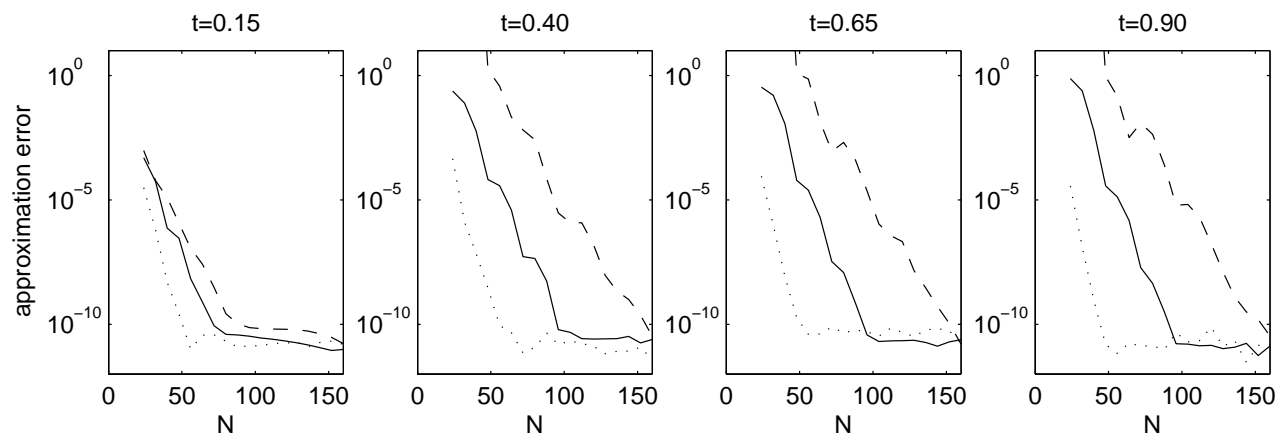

FIG. 5. Log-linear plots of approximation error against $N$ for the solution of (5.3) approximated using the adaptive rational spectral method. Dotted lines correspond to $\nu=10^{-2}$, solid lines to $\nu=10^{-3}$, and dashed lines to $\nu=10^{-4}$.

$\nu$. As $\nu$ decreases geometrically by a factor of 10 , the number of grid points required for the method to produce results accurate to the limit of temporal errors increases algebraically by about 50 .

6. Conclusion. We have presented an adaptive rational spectral method which far outperforms the Chebyshev spectral method for problems whose solutions have singularities in the complex plane close to $[-1,1]$. It combines ideas from existing rational spectral methods $[1,7]$ with an adaptive technique for locating singularities [25] using Chebyshev-Padé approximation. One could develop an analogous method for problems on periodic intervals. We are in the process of investigating further improvements of this method, as well as application to some of the innumerable problems of scientific computing whose solutions involve fronts, boundary layers, or other singularities.

\section{REFERENCES}

[1] R. Baltensperger and J.-P. Berrut, The linear rational collocation method, J. Comput. Appl. Math., 134 (2001), pp. 243-258.

[2] R. BALtensperger, J.-P. BerRut, AND B. NoËL, Exponential convergence of a linear rational interpolant between transformed Chebyshev points, Math. Comp., 68 (1999), pp. 1109-1120.

[3] Z. Battles And L. N. Trefethen, An extension of MATLAB to continuous functions and operators, SIAM J. Sci. Comput., 25 (2004), pp. 1743-1770.

[4] A. Bayliss, D. Gottlieb, B. Matkowsky, And M. Minkoff, An adaptive pseudospectral method for reaction diffusion problems, J. Comput. Phys., 81 (1989), pp. 421-443.

[5] A. Bayliss and B. Matkowsky, Fronts, relaxation oscillations and period doubling in solid fuel combustion, J. Comput. Phys., 71 (1987), pp. 147-188.

[6] A. Bayliss And E. TURKel, Mappings and accuracy for Chebyshev pseudo-spectral approximations, J. Comput. Phys., 101 (1992), pp. 349-359.

[7] J.-P. BerRut AND R. BALtensPeRger, The linear rational pseudospectral method for boundary value problems, BIT, 41 (2001), pp. 868-879.

[8] J.-P. Berrut, R. Baltensperger, and H. D. Mittelmann, Recent developments in barycentric rational interpolation, Internat. Ser. Numer. Math., 151 (2005), pp. 27-51.

[9] J.-P. Berrut and H. D. Mittelmann, Rational interpolation through the optimal attachment of poles to the interpolating polynomial, Numer. Algorithms, 23 (2000), pp. 315-328.

[10] - The linear rational pseudospectral method with iteratively optimized poles for two-point boundary value problems, SIAM J. Sci. Comput., 23 (2001), pp. 961-975.

$[11]+$ Linear rational interpolation and its application in approximation and boundary value problems, Rocky Mountain J. Math., 32 (2002), pp. 527-544. 
[12] - Optimized point shifts and poles in the linear rational pseudospectral method for boundary value problems, J. Comput. Phys., 204 (2005), pp. 292-301.

[13] J.-P. Berrut and L. N. Trefethen, Barycentric Lagrange interpolation, SiAM Rev., 46 (2004), pp. 501-517.

[14] D. M. DAY AND L. Romero, Roots of polynomials expressed as orthogonal polynomials, SIAM J. Numer. Anal., to appear (2005).

[15] J. W. DoLD, Analysis of the early stage of thermal runaway, Quart. J. Mech. Appl. Math., 38 (1985), pp. 361-387.

[16] J. R. Dormand and P. J. Prince, A family of embedded Runge-Kutta formulae, J. Comput. Appl. Math., 6 (1980), pp. 19-26.

[17] H. Guillard and R. Peyret, On the use of spectral methods for the numerical solution of stiff problems, Comput. Methods Appl. Mech. Engrg., 66 (1988), pp. 17-43.

[18] W. HuAng, Y. Ren, AND R. D. Russell, Moving mesh methods based on moving mesh partial differential equations, J. Comput. Phys., 113 (1994), pp. 279-290.

[19] D. Kosloff and H. Tal-Ezer, A modified Chebyshev pseudospectral method with an $O\left(N^{-1}\right)$ time step restriction, J. Comput. Phys., 104 (1993), pp. 457-469.

[20] A. A. LACEY, Diffusion models with blow-up, J. Comput. Appl. Math., 97 (1998), pp. 39-49.

[21] L. S. Mulholland, W. Huang, And D. M. Sloan, Pseudospectral solution of near-singular problems using numerical coordinate transformations based on adaptivity, SIAM J. Sci. Comput., 19 (1998), pp. 1261-1289.

[22] C. Schneider And W. Werner, Some new aspects of rational interpolation, Math. Comp., 47 (1986), pp. 285-299.

[23] S. P. SuETIN, On the convergence of rational approximations to polynomial expansions in domains of meromorphy of a given function., Math. USSR-Sb., 34 (1978), pp. 367-381.

[24] L. N. Trefethen, Spectral methods in MATLAB, SIAM, Philadelphia, 2000.

[25] J. A. C. Weideman, Computing the dynamics of complex singularities of nonlinear PDEs, SIAM J. Appl. Dyn. Syst., 2 (2003), pp. 171-186. 